\title{
Cellular Protrusions - Lamellipodia, Filopodia, Invadopodia and Podosomes - and their Roles in Progression of Orofacial Tumours: Current Understanding
}

\author{
Kamila Mohamed Om Alblazi*, Chong Huat Siar
}

\begin{abstract}
Background: Protrusive structures formed by migrating and invading cells are termed lamellipodia, filopodia, invadopodia and podosomes. Lamellipodia and filopodia appear on the leading edges of migrating cells and function to command the direction of the migrating cells. Invadopodia and podosomes are special F-actin-rich matrix-degrading structures that arise on the ventral surface of the cell membrane. Invadopodia are found in a variety of carcinomatous cells including squamous cell carcinoma of head and neck region whereas podosomes are found in normal highly motile cells of mesenchymal and myelomonocytic lineage. Invadopodia-associated protein markers consisted of 129 proteins belonging to different functional classes including WASP, NWASP, cortactin, Src kinase, Arp 2/3 complex, MT1-MMP and F-actin. To date, our current understanding on the role(s) of these regulators of actin dynamics in tumors of the orofacial region indicates that upregulation of these proteins promotes invasion and metastasis in oral squamous cell carcinoma, is associated with poor/worst prognostic outcome in laryngeal cancers, contributes to the persistent growth and metastasis characteristics of salivary gland adenoid cystic carcinoma, is a significant predictor of increased cancer risk in oral mucosal premalignant lesions and enhances local invasiveness in jawbone ameloblastomas.
\end{abstract}

Keywords: Lamellipodia - filopodia - invadopodia - podosomes

Asian Pac J Cancer Prev, 16 (6), 2187-2191

\section{Introduction}

The purpose of this review is to present the current understanding of cellular protrusions that promote diverse processes of tumor cell migration and invasion specifically in the orofacial region. The rationale was to provide some insights into their biology, regulation and in vivo relevance. This review also examined how these cellular protrusions vary in their names as well as in their microstructural compositions and precise function(s) in tumor cells, starting from their positions in the tumor cells to their roles in the invasion process. Four main types of these cellular structures would be discussed. They are filopodia, lamellipodia, invadopodia and podosomes. Podosomes and invadopodia are collectively known as invadosomes (Génot and Gligorijevic, 2014). They are regarded as the founding members of the invadosome family (Saltel et al., 2011). The formation and regulatory activities of these cellular 'feet' are governed by a variety of molecules that have specific roles in these structures. Among these regulators of actin dynamics are WASP, NWASP, cortactin, Src kinase, Arp 2/3, MT1-MMP and F-actin. In the ensuing review, accumulating evidence on the putative role(s) for each of these cellular structures in orofacial neoplasms and how each of these regulatory proteins modulate the actin assembly/disassembly in these structures during oral tumorigenesis and tumor invasion processes are presented.

\section{Cellular Protrusions: General Understanding}

Cell migration is a tightly coordinated multistep mechanism essential in many physiological processes such as embryogenic morphogenesis, immune surveillance, tissue repair and regeneration, while its aberrant regulation drives disease progression such as cancer invasion and metastasis (Condeelis and Segall, 2003). Protrusive structures formed by migrating and invading cells are termed filopodia, lamellipodia, podosomes and invadopodia based on their morphological, structural, and functional characteristics. Formation of these structures is driven by spatially- and temporally-regulated actin polymerization at the leading edge of these migratory cells (Yamaguchi and Condeelis, 2007)

\section{Lamellipodia}

Lamellipodia are defined as flat broad membranous protrusions located at the leading edge of the migrating cells. These protrusions purportedly are responsible for driving the migrating cells, by attaching and pulling the 
cell body (Yamaguchi and Condeelis, 2007). Lamellipodia determine the cell movement direction by protrusive force that arises from the actin network. This network is formed as a result of actin filament polymerization against the plasma membrane (Bryce et al., 2005). The complex organization within the lamellipodia consists of orthogonal arrays of actin filaments with branched actin filaments near the leading edge (Sureneni et al., 2012).

\section{Filopodia}

Filopodia are thin, finger-like projections formed by parallel bunches of actin filaments (Sureneni et al., 2012). As with lamellipodia, filopodia appear as spikelike processes at the leading edge of the migrating cells. Similar to lamellipodia, filopodia function to command the direction of the migrating cells and contribute to cancer cell invasion (Bryce et al., 2005; Stevenson et al., 2012).

\section{Podosomes}

Podosomes were first discovered in the 1980s and were initially described as adhesive structures in Rous sarcoma virus-transformed fibroblasts (David-Pfeuty and Singer, 1980) but were later found spontaneously in normal highly motile cells of myelomonocytic lineage (Sibony-Benyamini and Gil-Henn, 2012). Current views suggest podosomes are structures found in both normal and pathologic cells. Podosomes have also been demonstrated in a variety of cell types that were subjected to stimulation under cell stress or matrix receptor (Saltel et al., 2011). They are small protrusions measuring only $0.4 \mu \mathrm{m}$ in length and $1 \mu \mathrm{m}$ in diameter (Sibony-Benyamini and Gil-Henn, 2012). These cellular processes are specific F-actin rich protrusions created on the inner cell membrane of the migrating cells at points of cell-extracellular matrix (ECM) contacts. They consist of columnar arrays of actin filaments surrounding a narrow tubular invagination of plasmalemma roughly perpendicular to the substratum (Spinardi et al., 2004). These adhesive structures endow the cells with invasive capabilities (Spinardi et al., 2004; Linder, 2007; Génot and Gligorijevic, 2014). Under pathologic circumstances, podosomes in normal cells are suspected to facilitate the tumour cell invasion process (Stylli et al., 2008).

\section{Invadopodia}

Invadopodia are a special F-actin protrusions that arise on the internal surface of cell membrane and are closely located to the ECM (Sibony-Benyamini and Gil-Henn, 2012). They are finger-like ventral membrane protrusions form by cancer cells during migration (Buccione et al., 2004; Destaing et al., 2011). The length of the invadopodium may extend up to $2 \mu \mathrm{m}$ or more, whilst the diameter may vary from $0.1 \mu \mathrm{m}$ to 0.8 $\mu \mathrm{m}$. These protrusions are matrix-degrading structures involved in ECM proteolysis (Artym et al., 2006). This fact is considered an important discovery in molecular and cellular basis of tumour invasion (Stylli et al., 2008). These microstructural processes are composed of a group of protein-like adhesive molecules, membrane signaling and remodeling proteins, actin and actin regulatory proteins and matrix degradation enzymes (Yamaguchi and Condeelis, 2007 ;Destaing et al., 2011). Invadopodiaassociated protein markers consisted of 129 proteins belonging to different functional classes, including aerobic glycolysis and other metabolic pathways, protein synthesis, degradation and folding, cytoskeleton components and membrane-associated protein (Ayala et al., 2008). These markers are proteins are responsible for tumour metastases, inflammation and development. Each tumour invasion stage is executed by a group of proteins including proteases, integrins, GTpases, kinases and cytoskeleton interacting proteins. These proteins are involved in actin polymerization, cell signalling, membrane trafficking,ECM adhesion, and ECM degradation (Sibony-Benyamini and Gil-Henn, 2012). Among them, cortactin, NWASP and WIP are essential components that constitute core structure of invadopodia and these proteins are upregulated in cells with a migratory phenotype (Destaing et al., 2011).

\section{Cellular Protrusions: Regulatory Molecules and Biomarkers}

\section{F-actin}

Actin is one of the most abundant proteins and forms the essential building blocks of the cytoskeleton in mammalian cells (Stevenson et al., 2012). The actin filaments are assembled to form complex networks by more than 100 different types of actin-binding proteins, and these superstructures undergo constant remodelling. The role of actin cytoskeleton in various physiologic as well as pathologic processes is significant. It maintains cell shape and creates a physical force for cell division and migration. F-actin in many structures modulates important roles including formation of cell-cell junction in epithelial and endothelial cells, and in cell shape preservation. It acts with junctional actin in epithelial cells polarity development (Gagat et al., 2013).

There are some actin binding proteins which control the action of F-actin that are important for cell function and movement. One of these proteins is cofilin, which bind $\mathrm{F}$-actin to form a more tilted F-actin to increase its flexibility (Aihara and Oda, 2013). The F-actin and cofilin bind to form a new actin filament at the leading edge of

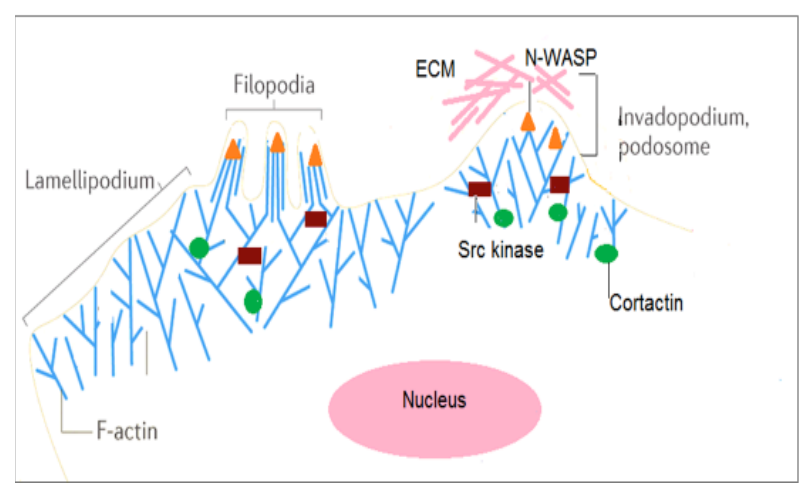

Figure 1. Schematic Diagram Showing the Various Types of Cellular Protrusions (Filopodia, Lamellipodium, Invadopodium and Podosome) and the Proteins (Cortactin, NWASP and Src-kinase) Involve in Regulating the Actin Network within the Tumor Cell 
the migrating cells to initiate the formation of membrane protrusions and promote the cell migration direction. This eventually results in the formation of podosome/ invadopodium and lamellipodium (Yamaguchi and Condeelis, 2007).

\section{Cortactin}

Cortactin is an F-actin binding protein. It is a major multidomain protein, implicated in actin polymerization (Webb et al., 2006; Shvetsov et al., 2009). Cortactin concentrated at cytosolic dynamic actin where it binds Arp 2/3 complex, F-actin and some signalling molecules such as src kinases, polyproline containing proteins as WIP, N-WASP and dynamine and contact the cells via its actin active sites like in lamellipodia, podosomes, and invadopodia ( Weaver et al., 2002; Bryce et al ., 2005). It functions as a scaffold to regulate Arp 2/3-mediated actin polymerization in invadopodia formation, thereby aiding cell migration and endocytosis of many different cell types (Shvetsov et al., 2009). Cortactin is enriched in invadopodia in a complex with paxillin and protein kinase, and is therefore considered as an early marker for invadopodia. It is overexpressed in various types of cancers and enhances cell motility (Buday and Downward, 2007).

The principal function of cortactin is to mediate the branching of actin by localization of actin assembly sites (Bryce et al., 2005). Cortactin contributes to invadopodia initiation via degradation of the underlying matrix substrate. It is also essential for trafficking of key proteases such as matrix metalloproteinases (MMP)-2 and -9 in head and neck squamous cell carcinomas (Weaver, 2008). Generally, cortactin has a relation with tumour invasiveness as exemplified in breast cancer and head and neck tumours (Stylli et al., 2008).

\section{WASP}

WASP (Wiskott-Aldrich syndrome protein) and N-WASP (neural WASP). These proteins are a nucleation control factors and share in actin cytoskeleton rearrangement process. Both WASP and N-WASP have an important effect in cell morphology and podosomes formation. N-WASP was shown in macrophages and could replace the deficiency of WASP in some circumstances, as in podosome matrix degradation (Isaac et al., 2010).

The role of WASP family in actin polymerization acts through the Arp2/3 complex and they in turn are regulated by Src family kinases. WASP acts as an activator for nucleation and branching of actin filament. It also has a role in cell protrusion, migration and invasion (Weaver et al., 2002; Yamaguchi and Condeelis, 2007). N-WASP specifically, is necessary for epithelial cell invasion. Thus, it is engaged in invasive membrane protrusions formation. Generally, both WASP and N-WASP are involved in matrix degrading structures in invadopodia and podosomes (Yamaguchi and Condeelis, 2007).

\section{Src kinase}

Src kinase is one of a non-receptor tyrosine kinase and a member of SFKs family (src family kinase). Studies have shown that src-kinase plays a major role in multiple intracellular signal transduction pathways essential for various cellular processes such as cell growth, differentiation, cell adhesion and motility and has been engaged in regulation of cell survival and proliferation (Elsberger, 2013). In normal cells src-family tyrosine kinases remain inactive by tyrosine phosphorylation while in cancer cells, sfks are considered as oncogenic enzymes that have a significant role in the initiation and progression of some tumours (Baldwin et al., 2014). Src has been shown to interact with adhesion molecules as well as the regulatory molecules important for regulation of cytoskeleton remodeling. The inhibition of src activity leads to inhibition of tumour progression and metastasis. Members of the src family of non-receptor tyrosine kinases play a key role in regulation of invadopodia assembly (Hauck et al., 2002). It is known that src tyrosine kinase stimulates invadopodia formation in fibroblasts. It is involved in differentiation of $\mathrm{T}$ cells, myoblasts, osteoclasts and chondrocytes (Stylli et al., 2008; Seltana et al., 2013).

C-Src-kinase is a non-receptor tyrosine kinase (Hauck et al., 2002). It has been demonstrated that c-src is overexpressed or constitutively active in a variety of human tumours. Also it is associated with the invasive phonotype in both early and advanced solid tumours.

\section{MT1-MMP}

Membrane type 1-matrix metalloproteinase (MT1MMP) is one of proteins that control invadopodia formation and function in tumours (Shields et al., 2012). Studies have shown that MT1-MMP has a pronounced role in human cancers and show correlation with tumour stages, invasion and metastases and reduced overall survival of patients. The membrane associated MMP, MT1-MMP, plays a pivotal role in pericellular proteolysis of ECM substrates including type I, II and III collagens, fibrin and laminin. MT1-MMP also initiate proteolytic cascades by activating other MMPs such as pro-MMP-2, -8 and -13 . Thus it modulates cell migration, and tumour growth and dissemination.

\section{Cellular Protrusions and Orofacial Tumours}

In recent years, considerable research effort has been directed towards identifying novel and accurate biomarkers primarily for purposes of prognostication and as predictive parameters. The regulatory molecules driving invadopodia-mediated cellular migration and invasion represent one such area of rigorous research activity. The potential roles of these proteins in influencing the growth and metastasis characteristics of cancers from other sites such as the breast (Eckert and Yang, 2011; Hong et al., 2013), pancreas (Shields et al., 2012), lungs (Noh et al., 2013), and prostate (Nakane et al., 2012) have been well-investigated. In contrast, much less is known about the role of these mediators of actin dynamics regulating the development and progression of orofacial neoplasms.

Hwang et al. (2012) investigated the precise part of invadopodia and some of their protein markers in one of most common oral tumoures, sequamous cell carcinoma (SCC). They reported that F-actin and MMP have a 
Kamila Mohamed Om Alblazi and Chong Huat Siar

function in ECM degradation during SCC formation and invasion. Other reports stated that suppression of invadopodia formation by microRNA-375 can reduce head and neck squamous cell carcinoma invasion (Jimenez et al., 2014). In addition, it has been shown that cortactin overexpression is a significant predictor of increased cancer risk in oral mucosal premalignant lesions (De Vicente et al., 2012).

In salivary gland tumors, researchers have found that invadopodia identified by their positive protein expressions for actin, cortactin, MT1-MMP and MMP-9 have an influence on adenoid cystic carcinoma formation (Nascimento et al., 2010). Furthermore, in adenoid cystic carcinoma which is well-recognized for its aggressive and relentless growth characteristics, cortactin overexpression may contribute to its invasion and metastasis abilities (Greer et al., 2007).

A study on the role of invadopodia in ameloblastoma documented that cortactin and MMP1 expressions are enhanced in the tumor epithelium of ameloblastoma compared to the stroma, suggesting a contribution to local invasiveness of this neoplasm (Pinheiro et al, 2011).

\section{Conclusions and Perspectives}

In the present paper, we documented recent progress in our understanding on the process of cellular protrusion formation focussing on protein molecules that promote these processes mediating cellular invasion in orofacial tumors. Although tremendous strides have been made in this area, many key questions remain (Murphy and Courtneidge, 2011). The fundamental $/$ molecular differences between the various cellular protrusions are not fully known. It is unclear how a cell type in the normal and diseased state(s) switches from one type of cellular protrusion to another type. The exact mechanism(s) whereby these cellular protrusions promote tumour invasion and metastasis is also unknown.

In conclusion, although the fine mechanisms that modulate the assembly/disassembly of the actin cytoskeletal network is not fully understood, the formation of these actin-rich microstructural processes underpins metastasis which is the leading cause of death in cancer patients. Therefore an understanding of the mechanism that governs invadopodia formation and function is an essential step in the prevention of cancer spread. In this paper, we presented an overview on the four main cellular protrusions formed by normal and cancerous cells, the proteins that regulate the formation of these processes and their clinical relevance specifically in relation to invasion, metastasis and prognosis of orofacial neoplasms. Accumulating data indicate that silencing/inhibition of protein molecules regulating these cellular protrusions could represent a promising strategy to control invasion and metastasis characteristics of these cancers.

\section{Ackhowledgement}

This study was supported by the University of Malaya Postgraduate Research Grant Scheme (PS038-2013B), and Frontier Science Research Cluster (RG335-15AFR).

\section{References}

Aihara T, Oda, T (2013). Cooperative and non-cooperative conformational changes of F-actin induced by cofilin. Biochem Biophys Res Commun, 435, 229-33.

Ambrosio EP, Rosa FE, Domingues MA, et al (2011) Cortactin is associated with perineural invasion in the deep invasive front area of laryngeal carcinomas. Hum Pathol, 42, 1221-9.

Ammer AG, Weed SA (2008). Cortactin branches out: roles in regulating protrusive actin dynamics. Cell Motil Cytoskeleton, 65, 687-707.

Artym VV, Zhang Y, Seillier-Moiseiwitsch F, Yamada KM, Mueller SC (2006). Dynamic interactions of cortactin and membrane type 1 matrix metalloproteinase at invadopodia: defining the stages of invadopodia formation and function. Cancer Res, 66, 3034-43.

Ayala I, Baldassarre M, Giacchetti G, et al (2008). Multiple regulatory inputs converge on cortactin to control invadopodia biogenesis and extracellular matrix degradation. J Cell Sci, 121, 369-78.

Baldwin GS, Lio DS-S, Ferrand A, et al (2014). Activation of Src family tyrosine kinases by ferric ions. Biochim Biophys Acta, 1844, 487-96.

Bryce NS, Clark ES, Leysath JML, et al (2005). Cortactin promotes cell motility by enhancing lamellipodial persistence. Curr Biol, 15, 1276-85.

Buccione R, Caldieri G, Avala I (2009). Invadopodia: specialized tumor cells structures for the focal degradation of the extracellular matrix. Cancer Metastasis Rev, 28, 137-49.

Buccione R, Orth JD, McNiven, MA (2004). Foot and mouth: podosomes, invadopodia and circular dorsal ruffles. Nat Rev Mol Cell Biol, 5, 647-57.

Buday L, Downward J (2007). Roles of cortactin in tumor pathogenesis. Biochim Biophys Acta, 1775, 263-73.

Condeelis J, Segall, JE (2003). Intravital imaging of cell movement in tumours. Nat Rev Cancer, 3, 921-30.

David-Pfeuty T, Singer SJ (1980). Altered distributions of the cytoskeletal proteins vinculin and alpha-actinin in cultured fibroblasts transformed by Rous sarcoma virus. Proc Natl Acad Sci USA, 77, 6687-91.

de Vicente JC, Rosado P, Lequerica-Fernandez P, et al (2013). Focal adhesion kinase overexpression: correlation with lymph node metastasis and shorter survival in oral squamous cell carcinoma. Head Neck, 35, 826-30.

Destaing O, Block MR, Planus E, Albiges-Rizo C (2011). Invadosome regulation by adhesion signaling. Curr Opin Cell Biol, 23, 597-606.

Eckert MA, Yang J (2011). Targeting invadopodia to block breast cancer metastasis. Oncotarget, 2, 562-8

Elsberger B (2014). Translational evidence on the role of Src kinase and activated Src kinase in invasive breast cancer. Crit Rev Oncol Hematol, 89, 343-51.

Gagat M, Grzanka D, Izdebska M, et al (2014). Tropomyosin-1 protects endothelial cell-cell junctions against cigarette smoke extract through F-actin stabilization in EA.hy926 cell line. Acta Histochem, 116, 606-18.

García E, Jonesb GE, Macheskyc LM, Antóna IM (2012). WIP: WASP-interacting proteins at invadopodia and podosomes. Eur J Cell Biol, 9, 869-77.

Génot E, Gligorijevic B (2014). Invadosomes in their natural habitat. Eur J Cell Biol, 93, 367-79.

Greer RO Jr, Said S, Shroyer KR, Marileila VG, Weed SA (2007). Overexpression of cyclin D1 and cortactin is primarily independent of gene amplification in salivary gland adenoid cystic carcinoma. Oral Oncol, 43, 735-41.

Grigera PR, Ma L, Borgman CA, et al (2012). Mass spectrometric analysis identifies a cortactin -RCC2/TD60 interaction in 
mitotic cells. J Proteomics, 75, 2153-9.

Hauck CR, Hsia DA, Ilic D, Schlaepfer DD (2002) v-Src SH3enhanced interaction with focal adhesion kinase at beta 1 integrin-containing invadopodia promotes cell invasion. $J$ Biol Chem, 277, 12487-90.

Hong BH, Wu CH, Yeh CT, Yen GC (2013). Invadopodiaassociated proteins blockade as a novel mechanism for 6-shogaol and pterostilbene to reduce breast cancer cell motility and invasion. Mol Nutr Food Res, 57, 886-95.

Hwang YS, Park KK, Chung WY (2012). Invadopodia formation in oral squamous cell carcinoma: the role of epidermal growth factor receptor signalling. Arch Oral Biol, 57, 335-43

Isaac BM, Ishihara D, Nusblat LM, et al (2010). N-WASP has the ability to compensate for the loss of WASP in macrophage podosome formation and chemotaxis. Exp Cell Res, 316, 3406-16.

Jimenez L, Sharma, VP, Lim J, et al (2014). MicroRNA-375 impairs head and neck squamous cell carcinoma invasion by suppressing invadopodia activity. Cancer Res, 74, 1452.

Linder S (2007). The matrix corroded: podosomes and invadopodia in extracellular matrix degradation. Trends Cell Biol, 17, 107-17.

Murphy DA, Courtneidge SA (2011). The 'ins' and 'outs' of podosomes and invadopodia: characteristics, formation and function. Nat Rev Mol Cell Biol, 12, 413-26.

Nascimento CF, Gama-De-Souza LN, Freitas VM, Jaeger RG (2010). Role of MMP9 on invadopodia formation in cells from adenoid cystic carcinoma. Study by laser scanning confocal microscopy. Microsc Res Tech, 273, 99-108.

Nakane K, Fujita Y, Terazawa R, et al (2012). Inhibition of cortactin and SIRT1 expression attenuates migration and invasion of prostate cancer DU145 cells. Int J Urol, 19, 71-9.

Noh SJ, Baek HA, Park HS, et al (2013). Expression of SIRT1 and cortactin is associated with progression of non-small cell lung cancer. Pathol Res Pract, 209, 365-70.

Pinheiro JJV, Nascimento CF, Freitas VM, et al (2011). Invadopodia proteins, cortactin and membrane type I matrix metalloproteinase (MT1-MMP) are expressed in ameloblastoma. Histopathol, 59, 1261-79.

Saltel F, Daubon T, Juin A, et al (2011). Invadosomes: intriguing structures with promise. Eur J Cell Biol, 90, 100-7.

Seano G, Daubon T, Génot E, Primo L (2014). Podosomes as novel players in endothelial biology. Eur J Cell Biol, 93, 405-12.

Seltana A, Guezguez A, Lepage, M, Basora, N, Beaulieu, J-F (2013). Src family kinase inhibitor PP2 accelerates differentiation in human intestinal epithelial cells. Biochem Biophys Res Commun, 430, 1195-200.

Shields MA, Krantz SB, Bentrem DJ, Dangi-Garimella S, Munshi HG (2012). Interplay between $\beta 1$-integrin and Rho signaling regulates differential scattering and motility of pancreatic cancer cells by snail and Slug proteins. J Biol Chem, 287, 6218-29.

Shvetsov A, Berkane E, Chereau D, Dominguez R, Reisler E (2009). The actin-binding domain of cortactin is dynamic and unstructured and affects lateral and longitudinal contacts in F-actin. Cell Motil Cytoskeleton, 66, 90-8.

Sibony-Benyamini H, Gil-Henn H (2012). Invadopodia: The leading force. Eur J Cell Biol, 91, 896-901.

Spinardi L, Rietdorf J, Nitsch L, et al (2004). A dynamic podosome-like structure of epithelial cells. Exp Cell Res, 295, 360-74.

Stevenson RP, Veltman D, Machesky LM (2012). Actin-bundling proteins in cancer progression at a glance. $J$ Cell Sci, 125, 1073-9.

Stylli SS, Kaye, AH, Lock P (2008). Invadopodia: At the cutting edge of tumour invasion. J Clin Neurosci, 15, 725-37.
Suraneni P, Rubinstein B, Unruh JR, et al (2012). The Arp2/3 complex is required for lamellipodia extension and directional fibroblast cell migration. J Cell Biol, 197, 239-51.

Weaver AM (2008). Cortactin in tumor invasiveness. Cancer Lett, 265, 157-66.

Weaver AM, Heuser JE, Karginov AV, et al (2002). Interaction of cortactin and N-WASP with Arp2/3 complex. Curr Biol, 12, 1270-8.

Webb BA, Eves R, Mak AS (2006). Cortactin regulates podosome formation: roles of the protein interaction domains. Exp Cell Res, 312, 760-9.

Yamada S, Yanamoto S, Kawasaki G, Mizuno A, Nemoto TK (2010). Overexpression of cortactin increases invasion potential in oral squamous cell carcinoma. Pathol Oncol Res, 16, 523-31.

Yamaguchi H, Condeelis, J (2007). Regulation of the actin cytoskeleton in cancer cell migration and invasion. Biochim Biophys Acta, 1773, 642-52. 